\title{
T-spherical fuzzy power aggregation operators and their applications in multi-attribute decision making
}

\author{
Harish Garg ${ }^{1}\left[\right.$ [D $\cdot$ Kifayat Ullah ${ }^{2} \cdot$ Tahir Mahmood $^{3} \cdot$ Nasruddin Hassan $^{4} \cdot$ Naeem Jan $^{3}$
}

Received: 16 September 2019 / Accepted: 3 October 2020 / Published online: 22 January 2021

○) Springer-Verlag GmbH Germany, part of Springer Nature 2021

\begin{abstract}
The paper aims to present the concept of power aggregation operators for the T-spherical fuzzy sets (T-SFSs). T-SFS is a powerful concept, with four membership functions denoting membership, abstinence, non-membership and refusal degree, to deal with the uncertain information as compared to other existing fuzzy sets. On the other hand, the relationship between the different pairs of the attributes are well recorded in terms of power operators. Thus, keeping these advantages of T-SFSs and power operator, the objective of this work is to define several weighted averaging and geometric power aggregation operators. The stated operators named as T-spherical fuzzy weighted, ordered weighted, hybrid averaging and geometric operators for the collection of the T-SFSs. The various properties and the special cases of them are also derived. Further, the consequences of proposed new power aggregation operators are studied in view of some constraints. Finally, a multiple attribute decision making algorithm, based on the proposed operators, is established to solve the problems with uncertain information and illustrate with numerical examples. A comparative study, superiority analysis and discussion of the proposed approach are furnished to confirm the approach.
\end{abstract}

Keywords T-spherical fuzzy sets $\cdot$ Power aggregation operators $\cdot$ Multi-attribute decision making $\cdot$ Membership degrees

\section{Introduction}

Multiple attribute decision making (MADM) process includes the examination of a limited arrangement of options and positioning them as far as the fact that they are so trustworthy to decision-maker(s) when all the rules are thought of at the same time. In this procedure, the rating estimations of every option incorporate both exact information

Harish Garg

harishg58iitr@gmail.com; harish.garg@thapar.edu

https://sites.google.com/site/harishg58iitr/home

Kifayat Ullah

kifayat.ullah@riphah.edu.pk

1 School of Mathematics, Thapar Institute of Engineering and Technology, Deemed University, Patiala, Punjab 147004, India

2 Department of Mathematics, Riphah Institute of Computing and Applied Sciences, Riphah International University Lahore, Lahore 54000, Pakistan

3 Department of Mathematics and Statistics, International Islamic University Islamabad, Islamabad, Pakistan

4 School of Mathematical Sciences, Faculty of Science and Technology, Universiti Kebangsaan Malaysia, 43600 Bangi, Malaysia and specialists' subjective data. However, generally, it is expected that the data gave by them are fresh in nature. In any case, because of the unpredictability of the framework step by step, the genuine contains numerous MADM issues where the data is either ambiguous, lose or dubious in nature. To manage it, Atanassov (1986) developed the framework of intuitionistic fuzzy set (IFS) which described the degree of imprecision in terms of two functions known as membership and non-membership functions with a condition that their sum must not exceed 1. Atanassov's intuitionistic fuzzy model enhanced the concept of Zadeh's fuzzy set (FS) (Zadeh 1965) which described the imprecision in an uncertain event with the help of only one function known as membership function on a scale of 0 to 1 . Atanassov's IFS restricts decision-makers in two ways; first, it restricts the sum of membership and non- membership grades up to unit interval due to which decision makers cannot assign these grades by their own consent. Second, it fails to be applied to those problems where opinion is not of yes or no type, but it has abstinence as well as refusal degree. However, in sequence to negotiate the ambiguities in the data, theories such as interval-valued IFS (IVIFS) (Atanassov and Gargov 1989), cubic intuitionistic fuzzy set (Garg and Kaur 2019), Pythagorean fuzzy set (PyFS) (Yager 2013), picture fuzzy 
set (PFS) (Cuong 2014), linguistic interval-valued IFS (Garg and Kumar 2019), linguistic interval-valued PyFS (Garg 2020b), q-rung orthopair fuzzy set (q-ROPFS) (Yager 2017) are used by the researchers. The benefits of such elongated extensions are that they serve uncertain information using membership, non-membership and the degree of hesitancy.

Since, the domain of Atanassov's IFS is very narrow which could not be applied to situations where opinion could be of more than two types such as yes, abstain, no and refusal. For this purpose, Cuong (2014) introduced a generalized model in FS theory known as picture fuzzy set (PFS) which describe four aspects of an imprecise event. In decision making, human opinion can be favor, abstinence, and disfavor and refusal degree represented by four membership functions of a PFS. Like Atanassov's IFS, Cuong's PFS has a restriction that the sum of three membership grades must not exceed 1 which somehow restricts the decision maker from setting up the membership values by their own consent. Mahmood et al. (2018) proposed a solution for the limited structure of PFS and developed the framework of spherical fuzzy set (SFS) by increasing the range of PFS. This concept of SFS is further extended to the idea of T-spherical fuzzy set (T-SFS) by introducing a parameter $t$ that allows the decision makers to choose the values of membership grades from anywhere in the interval [0,1]. A T-SFS is the most generalized fuzzy framework that can represent human opinion about any imprecise event in a flexible way with no limitations. The geometrical comparison between the ranges of PFS, SFS and T-SFS is portrayed in Fig. 1 which supports the superiority of T-SFS over other fuzzy frameworks.

MADM is a hot research area in fuzzy mathematics. It is regarded as one of the most influential topics that are discussed in almost every fuzzy framework. The commonly known tools for MADM process are the aggregation operators and in some cases distance, similarity measures. Several aggregation operators have been developed so far under the different environment. For example, under the IFS and PyFS environment, authors in (Xu 2007; Xu and Yager 2006) presented some weighted

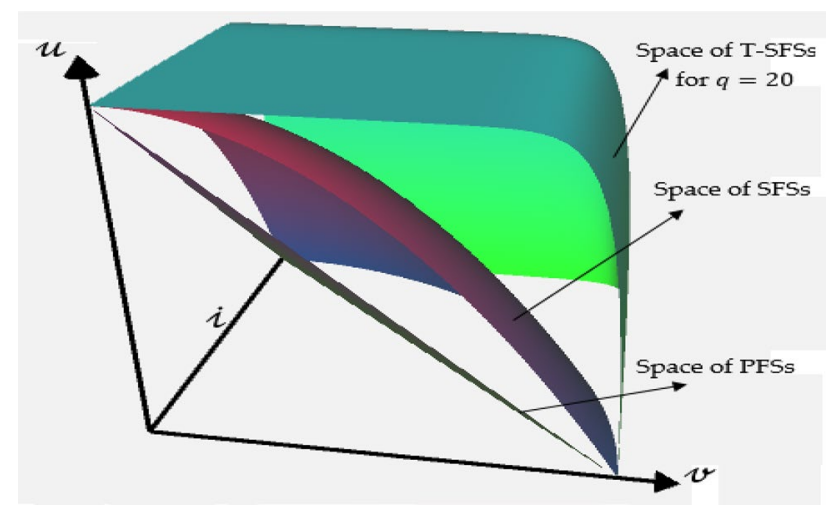

Fig. 1 Comparison of range of T-SFS with other PFS and SFS averaging (WA) and weighted geometric (WG) operators to aggregate the information. Peng and Yang (2015) examined the new operations on PyFS like division, subtraction to find the superiority and inferiority with the help of ranking method in MADM problems. The idea of Pythagorean fuzzy TODIM (an acronym in Portuguese for interactive Multi-criteria Decision Making) approach to MADM is developed by Ren et al. (2016). In the work of Garg (2017a), author investigated some new generalized Pythagorean fuzzy information aggregation operators by combining Einstein operations and studied some of their desirable properties. Kaur and Garg (2019) presented some t-norm based operators for cubic IFS to solve the MADM problems. Yang et al. (2020c) used Heronian mean operators and designed a MADM algorithm for online shopping. However, under q-ROPFS environment, the WA and WG operators are defined by Liu and Wang (2018) and studied their applications in MADM. Yang et al. (2020a) further investigated the multiple heterogeneous relationships using MADM approach of q-rung orthopair fuzzy information. Under the PFS environment, Garg (2017b) defined the weighted averaging and geometric operators while Wang et al. (2017) presented some geometric operators to solve the decision-making problems. Mahmood et al. (2018) extended these operations to the spherical fuzzy set (SFS) while Ullah et al. (2019) presented such operators to the T-SFS. The practicality of these aggregation operators is discussed in MADM problems. In Yang et al. (2020b), authors investigated the selection of antivirus mask in light of COVID-19 pandemic using SFSs. Ullah et al. (2020b) defined the Hamacher aggregation operators for T-SFS. Munir et al. (2020) used Einstein hybrid aggregation operators based on T-SFSs for solving MADM problems. Garg et al. (2018) presented some improved interactive operators for T-SFSs. In Liu et al. (2019), authors have solved the MADM problem by define the Muirhead mean operators for the T-SFS information. However, in terms of information measures such as similarity and correlation, some algorithms are presented in the literature (Ullah et al. 2018, 2020a) to solve the pattern recognition and other problems.

All the above stated measures and operators have been greatly utilized to solve the MADM problems. However, it is observed from these studies that all these algorithms are stated under the assumption that the considered attributes are independent to each other. But it is quite obvious that in our real-life, all the considered attributes are directly impact on each other. For instance, consider a decision making problem related to purchase of house then their corresponding attributes or parameters such as locality, price, features are directly depend on each other. Hence, in the above stated algorithm, such features are not considered. In other words, all the above defined operators do not consider the relationship of the values being used. To overcome this difficulty, a concept of power aggregation operator has been defined by Yager (2001). Power aggregation operators significantly 
involve the relationship of information being aggregated. Due to the importance of power aggregation operators, several authors have addressed the various MADM problems by utilizing the power operator. For instance, $\mathrm{Xu}$ and Yager (2010) proposed some power WG aggregation operators in fuzzy environment. Wei et al. (2013) utilized fuzzy power aggregation operators to solve the MADM problem. Wei and Lu (2018) express the power WA and WG operators for PyFS features. Xu (2011) examined these power operators with IFS features. Garg and Kumar (2020) utilized the concept of the connection number under IFS environment to define the power geometric operators. Zhou et al. (2012) defined the generalized power aggregation operators for solving the group decision making problems. Wang et al. (2020) defined interactive power Hamacher operators with PyFS information to solve the problems. Wang and $\mathrm{Li}$ (2020) defined interactive power Bonferroni mean operators to solve the MADM problem under the PyFS environment. Garg and Arora $(2018,2019)$ defined some scaled prioritized interactive and Archimedean t-norm based operators to solve the MADM problems. Rani and Garg (2018) developed the concept of complex intuitionistic fuzzy power aggregation operators and their applications in MADM process.

With the increasing complexities in the system, it is perceived that the above defined literature and the extension of FS, T-SFS is one of the most promotable and useful tool to express the uncertainties in the data. On the other hand, by considering the importance of power an aggregation operator which takes into account the relationship of the information being aggregated, and the flexibilities of the T-SFS to describe the uncertain information in the data, it is observed that the existing power operators (Garg and Kumar 2020; Wang et al. 2020; Yager 2001; Xu and Yager 2010; Wei et al. 2013; Wei and Zhang 2019; Wang and Li 2020; Wei and Lu 2018; Garg and Arora 2018, 2019; Xu 2011) under the different environment could not handle the case where the information has abstinence and refusal membership grades along with membership and non-membership grades. To address it completely, in this paper, our aim is to introduce the concept of power operator for the T-SFSs. The primary objectives of the work are listed as follows.

1. A concept of T-SFS has been utilized to express the uncertainties in the data.

2. To develop several powers weighted operators to aggregate the collective information.

3. Some special cases of the proposed operators are deduced under the existing environment.

4. To establish an MADM method based on the proposed operators to solve the problems.

5. To show the significance and superiority of proposed power aggregation operators over existing power aggregation operators numerically.
The rest of the paper is summarized as. In Sect. 2, we briefly overview some basic concepts related to T-SFS. In Sect. 3, we propose the power weighted averaging and geometric aggregation operators for T-SFNs and investigates their properties. The consequences and the special cases of the proposed operators are investigated in Sect. 4. In Sect. 5, we established an algorithm to deal with MADM problems based on the proposed operators. A numerical example is discussed in Sect. 6 to demonstrate the stated algorithm while a comparative analysis is summarized in Sect. 7 with the existing studies. Finally, a concrete conclusion is given in Sect. 8.

\section{Preliminaries}

In this section, some basic notions about T-SFSs are reviewed.

Definition 1 (Mahmood et al. 2018) A T-SFS is defined in terms of three functions known as membership $(s)$, abstinence $(u)$ and non-membership $(d)$ function with a restriction that

$0 \leq s^{t}+u^{t}+d^{t} \leq 1$

for some $t \in \mathbb{Z}^{+}$and $t \geq 1$. Moreover, the term refusal degree is denoted by $r$ and obtained as

$r=\sqrt[t]{1-\left(s^{t}+u^{t}+d^{t}\right)}$

For convenience, the triplet $(s, u, d)$ is referred as T-spherical fuzzy number (T-SFN).

\section{Remark 1 A T-SFS becomes}

1. SFS; if $t$ is taken as 2. (By Mahmood et al. 2018)

2. PFS; if $t$ is taken as 1. (By Cuong 2014)

3. q-ROPFS; if $u$ is considered as zero. (By Yager 2017)

4. PyFS; if $u$ is considered as zero and $t$ is taken as 2. (By Yager 2013)

5. IFS; if $u$ is considered as zero and $t$ is taken as 1 . (By Atanassov 1986)

6. FS; if $u$ and $d$ are considered as zero and $t$ is taken as 1 . (By Zadeh 1965)

Remark 1 clearly establishes the superiority of T-SFS over the pre-existing concepts. A T-SFS is described in terms of four membership functions denoting membership, abstinence, non-membership and refusal degree. Therefore, it can describe any human opinion in a better way comparative to SFS, PFS, q-ROPFS, PyFS and IFS. In voting phenomena or human decision making, human opinions are based on four aspects as vote in favor, abstain, vote against 
and refused to vote. In such type of problems, the idea of T-SFS can successfully be utilized for better results. Further, the parameter $t$ in T-SFS allows the decision makers to select any value from the unit interval with no restrictions. Hence, the concept of T-SFS is more likely to be utilized in practical problems than other pre-existing fuzzy frameworks. The versatile nature of T-SFS over other fuzzy frameworks is geometrically demonstrated in Fig. 1 where the first shape shows the range of PFNs, second one demonstrates the range of SFNs while the third one shows the range of T-SFNs for $t=5$. Increasing the value of $t$ will increase the space of T-SFNs which makes it the most reliable and generalized fuzzy framework.

Now we discuss the concept of power aggregation operators and their significance. The idea of power aggregation operator was first proposed by Yager (2001) when he developed the concept of power WA aggregation operators in fuzzy environment. The reason to develop power aggregation operator is that all other aggregation tools do not consider the relationship of the information being used. Yager (2001) introduced power WA operators for FSs which leads $\mathrm{Xu}$ and Yager (2010) to propose power WG operators in fuzzy environment for aggregation purpose.

Definition 2 (Xu and Yager 2010) Let $\mathrm{T}_{i}(i=1,2,3, \ldots n)$ denote FSs. Then PWA and PWG operators are defined as:

$\operatorname{PWA}\left(\mathrm{T}_{1}, \mathrm{~T}_{2}, \mathrm{~T}_{3}, \ldots \mathrm{T}_{n}\right)=\frac{\bigoplus_{j=1}^{n}\left(w_{j}\left(1+\mathrm{S}_{3}\left(\mathrm{~T}_{j}\right)\right) \mathrm{T}_{j}\right)}{\sum_{j=1}^{n} w_{j}\left(1+\mathrm{S}_{3}\left(\mathrm{~T}_{j}\right)\right)}$

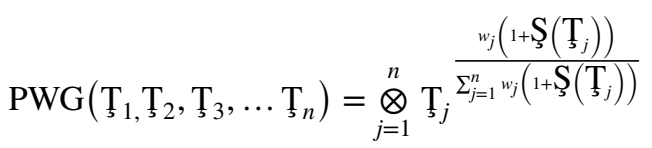

Motivated by the inspiring work on power aggregation operators in fuzzy environment by Yager (2001) and Xu and Yager (2010), Xu (2011) proposed the idea of power aggregation operators in the environment of IFS which was further extended by Wei and Lu (2018) to handle Pythagorean fuzzy information. The power aggregation operators developed by $\mathrm{Xu}$ (2011) for IFSs and by Wei and $\mathrm{Lu}$ (2018) for PyFSs are given as follows:

Definition 3 (Xu 2011) Let $T_{i}(i=1,2,3, \ldots n$ ) denote IFSs. Then PWA and PWG operators are defined as:

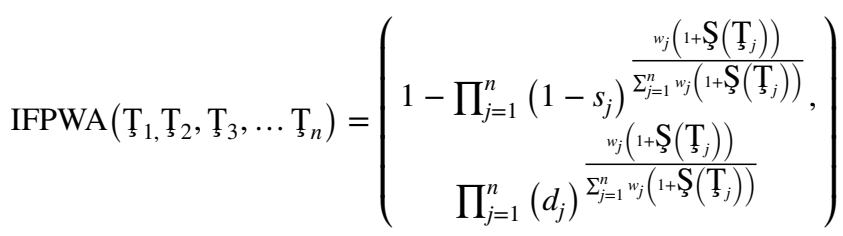

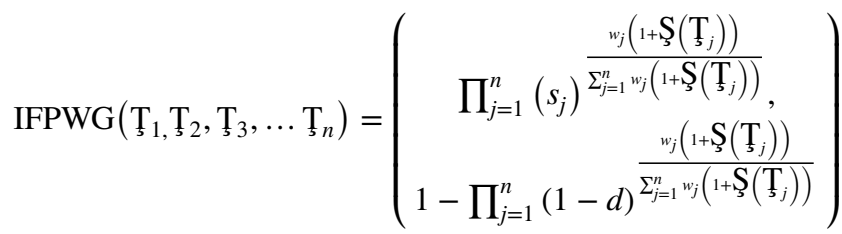

Definition 4 (Wei and Lu 2018) Let $\mathrm{T}_{i}$ denote some PyFSs. Then PWA and PWG operators are defined as:

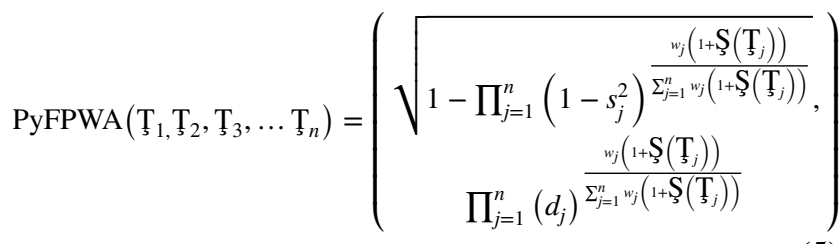

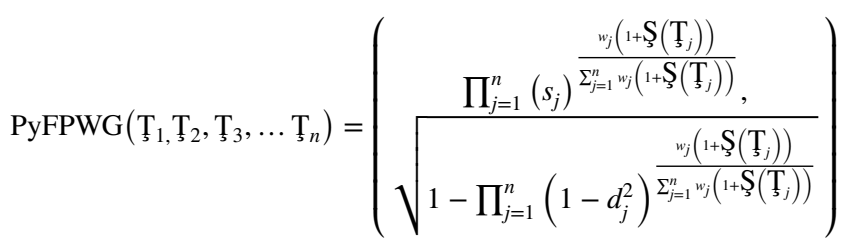

Keeping the significance of power aggregation operators and inspiring work in this direction, we proposed the power aggregation operators for T-SFSs and consequently for SFSs, PFSs and q-ROPFSs.

\section{Proposed Power aggregation operators for T-SFNs}

In this section, using the idea of power aggregation operators, we present the concept of power aggregation operator for T-SFSs and proposed TSFPWA operator, TSFPOWA operator, TSFPHA operator. The investigation of properties of the newly established concepts is carried out and their consequences are discussed. Throughout this paper, the weight vector is denoted by $w=\left(w_{1}, w_{2}, \ldots w_{n}\right)^{\frac{T}{3}}$ such that $w_{i}>0$ and $\sum_{1}^{n} w_{i}=1$. The terms $i, j$ and $k$ denote the indexing sets such that $i, j, k=1,2,3, \ldots n$. Let $\Gamma$ be a collection of T-SFNs.

Definition 4 Let $\mathrm{T}_{i}=\left(s_{i}, u_{i}, d_{i}\right)$ denote the collections of T-SFNs. Then TSFPWA operator is a map TSFPWA: $\Gamma^{\mathrm{n}} \rightarrow \Gamma$ defined as.

$\operatorname{TSFPWA}\left(\mathrm{T}_{1}, \mathrm{~T}_{2}, \ldots, \mathrm{T}_{n}\right)=\frac{\bigoplus_{j=1}^{n}\left(w_{j}\left(1+\mathrm{S}_{3}\left(\mathrm{~T}_{j}\right)\right) \mathrm{T}_{j}\right)}{\sum_{j=1}^{n} w_{j}\left(1+\mathrm{S}_{3}\left(\mathrm{~T}_{j}\right)\right)}$ 


$$
=\left(\begin{array}{c}
\sqrt[t]{1-\prod_{j=1}^{n}\left(1-s_{j}^{t}\right)^{\frac{w_{j}\left(1+\mathbf{S}_{j}\left(\mathbf{T}_{j}\right)\right)}{\sum_{j=1}^{n} w_{j}\left(1+\mathbf{S}_{3}\left(\mathbf{T}_{j}\right)\right)}}}, \\
\prod_{j=1}^{n}\left(u_{j}\right)^{\frac{w_{j}\left(1+\mathbf{S}_{j}\left(\mathbf{T}_{j}\right)\right)}{\sum_{j=1}^{n} w_{j}\left(1+\mathbf{S}_{3}\left(\mathbf{T}_{j}\right)\right)}} \prod_{j=1}^{n}\left(d_{j}\right)^{\frac{w_{j}\left(1+\mathbf{S}_{j}\left(\mathbf{T}_{j}\right)\right)}{\sum_{j=1}^{n} w_{j}\left(1+\mathbf{S}_{3}\left(\mathbf{T}_{j}\right)\right)}}
\end{array}\right)
$$

where $\quad \mathbf{S}_{(}\left(\mathrm{T}_{j}\right)=\sum_{\substack{i=1 \\ i \neq j}}^{n} w_{j} \operatorname{Sup}\left(\mathrm{T}_{j}, \mathrm{~T}_{i}\right)$ is the support function.

The aggregated operator defined in Definition 4 likely to satisfy the following basic properties of aggregation.

Property 1 (Monotonicity) Let $\mathrm{T}_{j}=\left(s_{\mathrm{T}_{j}}, u_{\mathrm{T}_{j}}, d_{\mathrm{T}_{j}}\right)$ and $\mathrm{P}_{j}=\left(s_{\mathrm{P}_{j}}, u_{\mathrm{P}_{j}}, d_{\mathrm{P}_{j}}\right)$ be two T-SFNs such that $\mathrm{T}_{j} \leq \mathrm{P}_{j} \forall j$. Then $\operatorname{TSFPWA}\left(\mathrm{T}_{1}, \mathrm{~T}_{2}, \ldots, \mathrm{T}_{n}\right) \leq \operatorname{TSFPWA}\left(\mathrm{P}_{1}, \mathrm{P}_{2}, \ldots, \mathrm{P}_{n}\right)$

Property 2 (Boundedness) If $\mathrm{T}^{-}=\left(\min _{j} s_{j}, \max _{j} u_{j}, \max _{j} d_{j}\right)$ and $\mathrm{T}^{+}=\left(\max _{j} s_{j}, \min _{j} u_{j}, \min _{j} d_{j}\right)$. Then

$\mathrm{T}^{-} \leq \operatorname{TSFPWA}\left(\mathrm{T}_{1}, \mathrm{~T}_{2}, \mathrm{~T}_{3} \ldots \mathrm{T}_{n}\right) \leq \mathrm{T}^{+}$

Property 3 (Idempotency) If $\forall j=1,2,3, \ldots n$, $\mathrm{T}_{j}=\mathrm{T}_{3}=(s, u, d)$. Then

$\operatorname{TSFPWA}\left(\mathrm{T}_{1}, \mathrm{~T}_{2}, \ldots, \mathrm{T}_{n}\right)=\mathrm{T}_{3}$

The TSFPWA operator defined in Definition 4 does not weigh the ordered position of T-SFNs but in only weigh the T-SFNs. There are many decision-making problems where the information is arranged in ascending or descending order and their ordered positions are weighted. To cope with such situation, inspired by the idea of Yager (2001), we proposed the concept of TSFPOWA operators in the following definition.

Definition 5 Let $\mathrm{T}_{i}=\left(s_{i}, u_{i}, d_{i}\right)$ denote some T-SFNs. Then TSFPOWA operator is defined as

$$
\begin{aligned}
& \operatorname{TSFPOWA}\left(\mathrm{T}_{1}, \mathrm{~T}_{2}, \ldots, \mathrm{T}_{n}\right)=\frac{\bigoplus_{j=1}^{n}\left(w_{j}\left(1+\mathrm{S}_{3}\left(\mathrm{~T}_{\sigma(j)}\right)\right) \mathrm{T}_{\sigma(j)}\right)}{\sum_{j=1}^{n} w_{j}\left(1+\mathrm{S}_{(}\left(\mathrm{T}_{\sigma(j)}\right)\right)}
\end{aligned}
$$

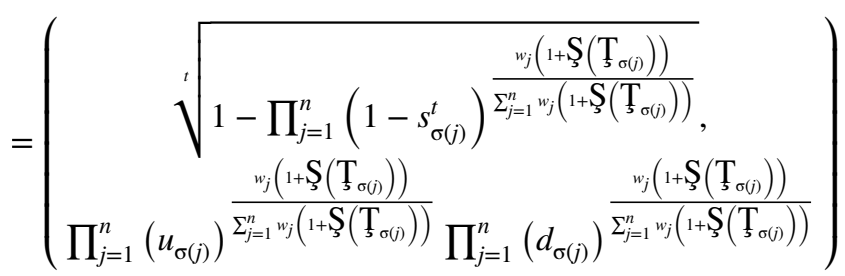

where $\sigma(j)$ is a permutation such that $\mathrm{T}_{\sigma(j-1)} \geq \mathrm{T}_{\sigma(j)} \forall j$ and $w_{j}$ denotes the collection of weights such that

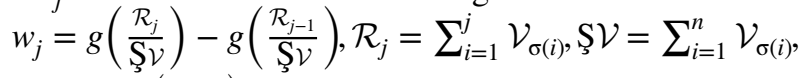

$$
\begin{aligned}
& \mathcal{V}_{\sigma(i)}=1+\operatorname{SS}_{\mathcal{G}}\left(\mathrm{T}_{\sigma(i)}\right) \text { and } \\
& \operatorname{S}\left(\mathrm{T}_{\sigma(j)}\right)=\sum_{\substack{i=1 \\
i \neq j}}^{n} \operatorname{Sup}\left(\mathrm{T}_{\sigma(j)}, \mathrm{T}_{\sigma(i)}\right)
\end{aligned}
$$

where $\mathrm{S}_{(}\left(\mathrm{T}_{\sigma(j)}\right)$ denote the support of $j$ th largest T-SFN $\mathrm{T}_{\sigma(j)}$ for $i$ th greatest T-SFN $\mathrm{T}_{\sigma(i)}$ and $g:[0,1] \rightarrow[0,1]$ is a monotonic function having the properties $g(0)=0, g(1)=1$ and $g(\mathrm{x}) \geq g(\mathrm{y})$ if $\mathrm{x}>y$.

The aggregated operator defined in Definition 5 likely to satisfy the following basic properties (1-3) of aggregation operators.

The TSFPWA operator weighs the argument only while the TSFPOWA operator weighs the ordered position of the argument instead i.e. both operators have two separate aspects. Therefore, in the following Definition, we proposed the concept of hybrid operator known as TSFPHA operator which weighs the argument as well as its ordered position.

Definition 6 Let $\mathrm{T}_{i}=\left(s_{i}, u_{i}, d_{i}\right)$ denote some T-SFNs. Then TSFPHA operator is defined as

$$
\begin{aligned}
& \operatorname{TSFPHA}\left(\dot{T}_{1}, \dot{T}_{2}, \ldots, \dot{T}_{n}\right) \\
& =\frac{\bigoplus_{j=1}^{n}\left(w_{j}\left(1+S_{S}\left(\dot{T}_{\sigma(j)}\right)\right) \dot{T}_{\sigma(j)}\right)}{\sum_{j=1}^{n} w_{j}\left(1+S_{\zeta}\left(\dot{T}_{\sigma(j)}\right)\right)}
\end{aligned}
$$

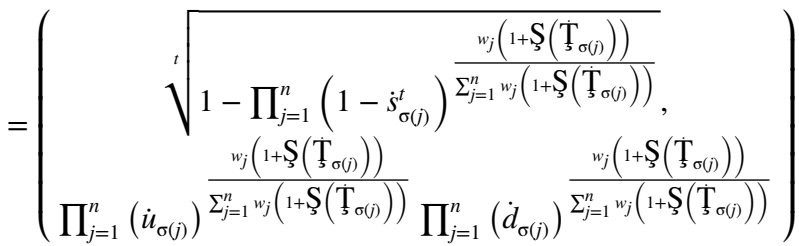

where $\dot{\mathrm{T}}_{\sigma(j)}$ is the $j$ th largest of the T-SFN $\dot{\mathrm{T}}_{j}=m \mathrm{w}_{j} \mathrm{~T}_{j}$ with $\mathrm{w}_{j}$ as the weight vector of T-spherical fuzzy arguments $\mathrm{T}_{j}$ such that $\mathrm{w}_{j} \in[0,1]$ and $\sum_{1}^{n} \mathrm{w}_{j}=1$ and $m$ is the balancing coefficient. Further, $w_{j}$ is such that

$$
\begin{aligned}
& w_{j}=g\left(\frac{\mathcal{R}_{j}}{\operatorname{Ş}_{\mathcal{V}}}\right)-g\left(\frac{\mathcal{R}_{j-1}}{\mathbf{S}_{\mathcal{V}}}\right), \mathcal{R}_{j}=\sum_{i=1}^{j} \mathcal{V}_{\sigma(i)}, \mathrm{S} \mathcal{V}=\sum_{i=1}^{n} \mathcal{V}_{\sigma(i)}, \\
& \left.\mathcal{V}_{\sigma(i)}=1+\dot{\mathrm{T}}_{\sigma(i)}\right) \text { and } \\
& \operatorname{SS}_{\left(\dot{\mathrm{T}}_{\sigma(j)}\right)}=\sum_{\substack{i=1 \\
i \neq j}}^{n} \operatorname{Sup}\left(\dot{\mathrm{T}}_{\sigma(j)}, \dot{\mathrm{T}}_{\sigma(i)}\right)
\end{aligned}
$$

where $\mathrm{S}_{(}\left(\dot{\mathrm{T}}_{\sigma(j)}\right)$ denote the support of $j$ th largest T-SFN $\dot{\mathrm{T}}_{\sigma(j)}$ for $i$ th greatest T-SFN $\dot{T}_{\sigma(i)}$ and $g:[0,1] \rightarrow[0,1]$ is a monotonic function having the properties $g(0)=0, g(1)=1$ and $g(\mathrm{x}) \geq g(\mathrm{y})$ if $\mathrm{x}>y$. 
Remark 2 Placing $w_{j}=\left(\frac{1}{n}, \frac{1}{n}, \frac{1}{n}, \ldots \frac{1}{n}\right)^{\mathrm{T}} \quad$ and $\mathrm{w}_{j}=\left(\frac{1}{\mathrm{n}}, \frac{1}{\mathrm{n}}, \frac{1}{\mathrm{n}}, \ldots \frac{1}{\mathrm{n}}\right)^{\mathrm{T}}$, the TSFPHA operator reduces to TSFPWA operator and TSFPOWA operator respectively.

The concept of power geometric aggregation operators is proposed by $\mathrm{Xu}$ and Yager (2010). We use the idea of power geometric aggregation operators developed by $\mathrm{Xu}$ and Yager (2010) and by using the operational laws developed by Ullah et al. (2019) to develop the concept of power geometric aggregation operators for T-SFSs and proposed TSFPG operator, TSFPOWG operator and TSFPHG operator. It is discussed that power geometric aggregation operators of T-SFSs satisfy the basic properties of aggregation including monotonicity, idempotency and boundedness. The consequences of the new proposed aggregation operators are also investigated.

Definition 7 Let $\mathrm{T}_{i}=\left(s_{i}, u_{i}, d_{i}\right)$ denote some T-SFNs. Then TSFPWG operator is defined as

$\operatorname{TSFPWG}\left(\mathrm{T}_{1}, \mathrm{~T}_{2}, \ldots, \mathrm{T}_{n}\right)=\bigotimes_{j=1}^{n} \mathrm{~T}_{j} \frac{w_{j}\left(1+\mathrm{S}_{j}\left(\mathrm{~T}_{j}\right)\right)}{\sum_{j=1}^{n} w_{j}\left(1+\mathrm{S}_{3}\left(\mathrm{~T}_{j}\right)\right)}$

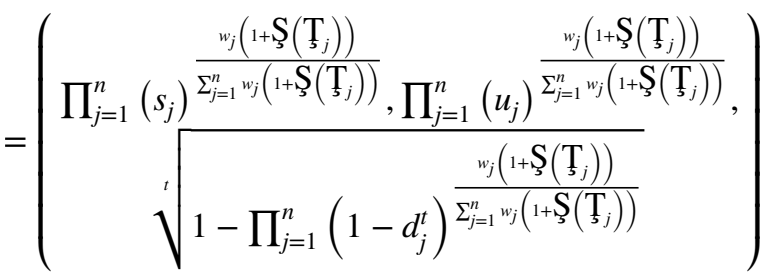

where

$\mathrm{S}_{3}\left(\mathrm{~T}_{s}\right)=\sum_{\substack{i=1 \\ i \neq j}}^{n} w_{j} \operatorname{Sup}\left(\mathrm{T}_{s}, \mathrm{~T}_{i}\right)$

The aggregated operator defined in Definition 7 likely to satisfy the following basic properties of aggregation.

Property 4 (Monotonicity) Let $\mathrm{T}_{j}=\left(s_{\mathrm{T}_{j}}, u_{\mathrm{T}_{j}}, d_{\mathrm{T}_{j}}\right)$ and $\mathrm{P}_{j}=\left(s_{\mathrm{P}_{j}}, u_{\mathrm{P}_{j}}, d_{\mathrm{P}_{j}}\right)$ be two T-SFNs such that $\mathrm{T}_{j} \leq \mathrm{P}_{j} \forall j$. Then

$\operatorname{TSFPWG}\left(\mathrm{T}_{1}, \mathrm{~T}_{2}, \mathrm{~T}_{3} \ldots \mathrm{T}_{n}\right) \leq \operatorname{TSFPWG}\left(\mathrm{P}_{1}, \mathrm{P}_{2}, \mathrm{P}_{3} \ldots \mathrm{P}_{n}\right)$

Property 5 (Boundedness) If $\mathrm{T}^{-}=\left(\min _{j} s_{j}, \max _{j} u_{j}, \max _{j} d_{j}\right)$ and $\mathrm{T}^{+}=\left(\max _{j} s_{j}, \min _{j} u_{j}, \min _{j} d_{j}\right)$. Then

$\mathrm{T}^{-} \leq \operatorname{TSFPWG}\left(\mathrm{T}_{1}, \mathrm{~T}_{2}, \mathrm{~T}_{3} \ldots \mathrm{T}_{n}\right) \leq \mathrm{T}^{+}$
Property 6 (Idempotency) If $\forall j=1,2,3, \ldots n$, $\mathrm{T}_{j}=\mathrm{T}_{s}=(s, u, d)$. Then

$\operatorname{TSFPWG}\left(\mathrm{T}_{1}, \mathrm{~T}_{2}, \mathrm{~T}_{3} \ldots \mathrm{T}_{n}\right)=\mathrm{T}_{3}$

Here again, the TSFPWG operator only weigh the T-spherical fuzzy argument but not its ordered position. Therefore, inspired by the idea of Xu and Yager (2010), the concept of TSFPOWG operator is proposed as follows.

Definition 8 Let $\mathrm{T}_{i}=\left(s_{i}, u_{i}, d_{i}\right)$ denote some T-SFNs. Then TSFPOWG operator is defined as

$\operatorname{TSFPOWG}\left(\mathrm{T}_{1}, \mathrm{~T}_{2}, \mathrm{~T}_{3}, \ldots \mathrm{T}_{3}\right)=\bigotimes_{j=1}^{n} \mathrm{~T}_{3} \frac{w_{j}\left(1+\mathrm{S}_{j}\left(\mathrm{~T}_{\sigma(j)}\right)\right)}{\sum_{j=1}^{n} w_{j}\left(1+\mathrm{S}_{3}\left(\mathrm{~T}_{\sigma(j)}\right)\right)}$

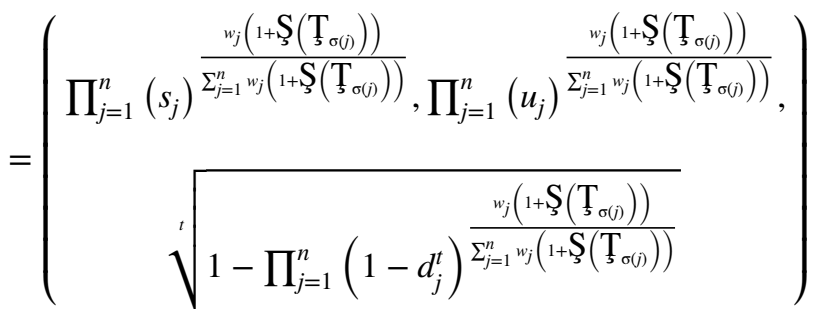

where $\sigma(j)$ is a permutation such that $\mathrm{T}_{\sigma(j-1)} \geq \mathrm{T}_{\sigma(j)} \forall j$ and $w_{j}$ denotes the collection of weights such that

$w_{j}=g\left(\frac{\mathcal{R}_{j}}{\mathbf{S}_{\mathcal{V}}}\right)-g\left(\frac{\mathcal{R}_{j-1}}{\mathbf{S}_{\mathcal{V}}}\right), \mathcal{R}_{j}=\sum_{i=1}^{j} \mathcal{V}_{\sigma(i)}, \mathbf{S} \mathcal{V}=\sum_{i=1}^{n} \mathcal{V}_{\sigma(i)}$, $\mathcal{V}_{\sigma(i)}=1+\mathrm{S}_{(}\left(\mathrm{T}_{\sigma(i)}\right)$ and

$\operatorname{S}\left(\mathrm{T}_{\sigma(j)}\right)=\sum_{\substack{i=1 \\ i \neq j}}^{n} \operatorname{Sup}\left(\mathrm{T}_{\sigma(j)}, \mathrm{T}_{\sigma(i)}\right)$

where $\mathrm{S}_{(}\left(\mathrm{T}_{\sigma(j)}\right)$ denote the support of $j$ th largest T-SFN $\mathrm{T}_{\sigma(j)}$ for $i$ th greatest T-SFN $\operatorname{T}_{\sigma(i)}$ and $g:[0,1] \rightarrow[0,1]$ is a monotonic function having the properties $g(0)=0, g(1)=1$ and $g(\mathrm{x}) \geq g(\mathrm{y})$ if $\mathrm{x}>y$.

The aggregated operator defined in Definition 8 likely to satisfy the following basic properties (4-6) of aggregation operators.

The TSFPWG operator weighs the T-spherical fuzzy argument only while the TSFPOWG operator weighs the ordered position of the argument instead. Therefore, we proposed the concept of TSFPHG operator which weighs the T-spherical fuzzy argument as well as its ordered position.

Definition 9 Let $\mathrm{T}_{i}=\left(s_{i}, u_{i}, d_{i}\right)$ denote some T-SFNs. Then TSFPHG operator is defined as 


$$
\begin{aligned}
& \operatorname{TSFPHG}\left(\dot{\mathrm{T}}_{1}, \dot{\mathrm{T}}_{2}, \dot{\mathrm{T}}_{3}, \ldots \dot{\mathrm{T}}_{n}\right)=\bigotimes_{j=1}^{n} \frac{\mathrm{T}_{j}}{\underline{w}_{j=1}^{n}\left(1+\mathrm{S}_{j}\left(\mathrm{~T}_{\sigma(j)}\right)\right)}
\end{aligned}
$$

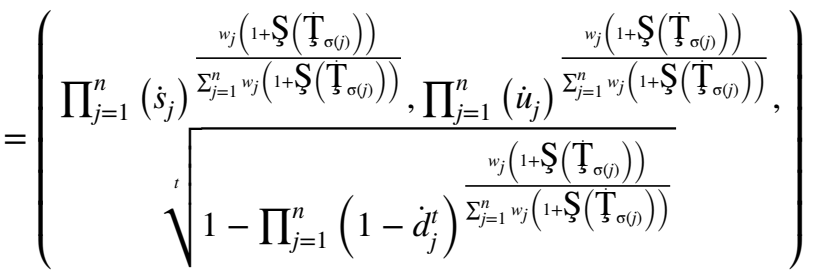

where $\dot{\mathrm{T}}_{\sigma(j)}$ is the $j$ th largest of the T-SFN $\dot{\mathrm{T}}_{j}=\mathrm{T}_{j}^{m \mathrm{w}_{j}}$ with $\mathrm{w}_{j}$ as the weight vector of T-spherical fuzzy arguments $\mathrm{T}_{j}$ such that $\mathrm{w}_{j} \in[0,1]$ and $\sum_{1}^{n} \mathrm{w}_{j}=1$ and $m$ is the balancing coefficient. Further, $w_{j}$ is such that

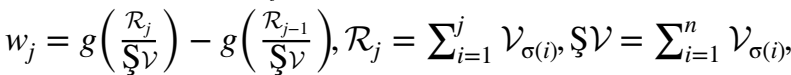

$$
\begin{aligned}
& \mathcal{V}_{\sigma(i)}=1+S_{S}\left(\dot{T}_{\sigma(i)}\right) \text { and } \\
& \operatorname{S}\left(\dot{\mathrm{T}}_{\sigma(j)}\right)=\sum_{\substack{i=1 \\
i \neq j}}^{n} \operatorname{Sup}\left(\dot{\mathrm{T}}_{\sigma(j)}, \dot{\mathrm{T}}_{\sigma(i)}\right)
\end{aligned}
$$

where $\left.\mathrm{S}_{\left(\mathrm{T}_{\sigma(j)}\right.}\right)$ denote the support of $j$ th largest T-SFN $\dot{\mathrm{T}}_{\sigma(j)}$ for $i$ th greatest T-SFN $\dot{\mathrm{T}}_{\sigma(i)}$ and $g:[0,1] \rightarrow[0,1]$ is a monotonic function having the properties $g(0)=0, g(1)=1$ and $g(\mathrm{x}) \geq g(\mathrm{y})$ if $\mathrm{x}>y$.

Remarks 3 Placing $w_{j}=\left(\frac{1}{\mathrm{n}}, \frac{1}{\mathrm{n}}, \frac{1}{\mathrm{n}}, \ldots \frac{1}{\mathrm{n}}\right)^{\mathrm{T}} \quad$ and $\mathrm{w}_{j}=\left(\frac{1}{\mathrm{n}}, \frac{1}{\mathrm{n}}, \frac{1}{\mathrm{n}}, \ldots \frac{1}{\mathrm{n}}\right)^{\mathrm{T}}$ reduces the TSFPHG operator to TSFPWG operator and TSFPOWG operator respectively.

\section{Consequences of the proposed work}

In this section, the consequences of theory developed in Sect. 3 are investigated which shows the superiority of proposed work over the pre-existing notions. Here using some restrictions, we show that proposed TSFPWA and TSFPWG operators are generalizations of power WA and power WG operators of IFSs and PyFSs studied in Wei and Lu (2018) and $\mathrm{Xu}$ (2011). Further, some restrictions on TSFPWA and TSFPWG aggregation operators also results in the development of power WA and power WG operators for SFSs, PFSs and q-ROPFSs.

Consider the following TSFPWA and TSFPWG operators labeled by Eqs. (7) and (10) respectively;
$\operatorname{TSFPWA}\left(\mathrm{T}_{1} \mathrm{~T}_{2}, \mathrm{~T}_{3}, \ldots \mathrm{T}_{n}\right)$

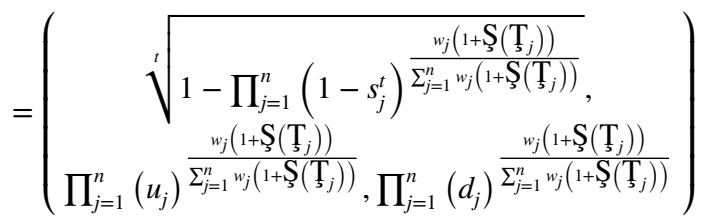

$\operatorname{TSFPWG}\left(\mathrm{T}_{1}, \mathrm{~T}_{2}, \mathrm{~T}_{3}, \ldots \mathrm{T}_{n}\right)$

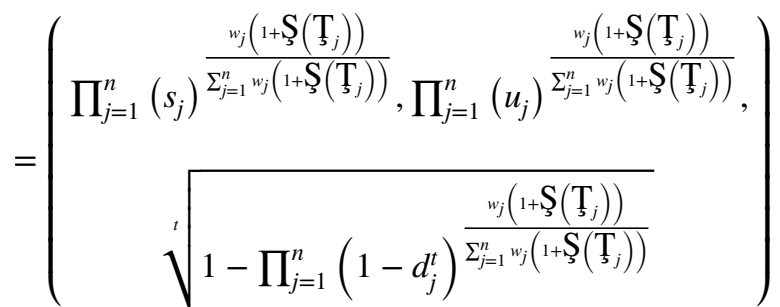

1. The Eqs. (7) and (10) results in the power WA and power WG aggregation operators of SFSs by taking $\mathrm{t}=2$ and given as:

$\operatorname{SFPWA}\left(\mathrm{T}_{1} \mathrm{~T}_{2}, \mathrm{~T}_{3}, \ldots \mathrm{T}_{n}\right)$

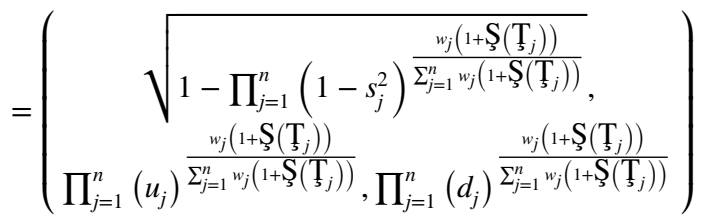

$$
\begin{aligned}
& \operatorname{SFPWG}\left(\mathrm{T}_{1}, \mathrm{~T}_{2}, \mathrm{~T}_{3}, \ldots \mathrm{T}_{n}\right)
\end{aligned}
$$

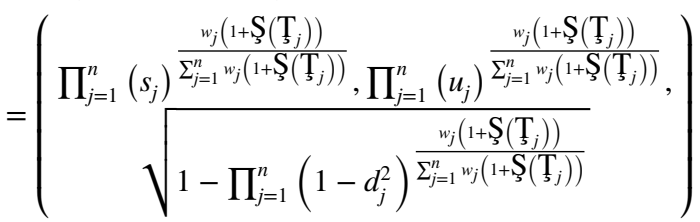

2. The Eqs. (7) and (10) results in the power WA and power WG aggregation operators of PFSs by taking $t=1$ and given as:

$$
\begin{aligned}
& \operatorname{PFPWA}\left(\mathrm{T}_{1}, \mathrm{~T}_{2}, \mathrm{~T}_{3}, \ldots \mathrm{T}_{n}\right)
\end{aligned}
$$

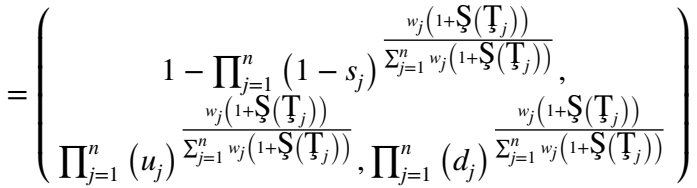




$$
\begin{aligned}
& \operatorname{PFPWG}\left(\mathrm{T}_{1}, \frac{\mathrm{T}_{2}}{3}, \mathrm{~T}_{3}, \ldots \mathrm{T}_{n}\right)
\end{aligned}
$$

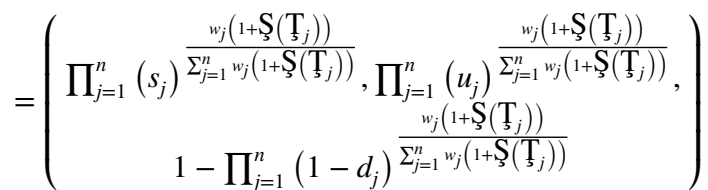

3. The Eqs. (7) and (10) results in the power WA and power WG aggregation operators of q-ROPFSs by taking $u_{j}=0$ and given as:

$\mathrm{q}-\operatorname{ROPFPWA}\left(\mathrm{T}_{1}, \mathrm{~T}_{2}, \mathrm{~T}_{3}, \ldots \mathrm{T}_{n}\right)$

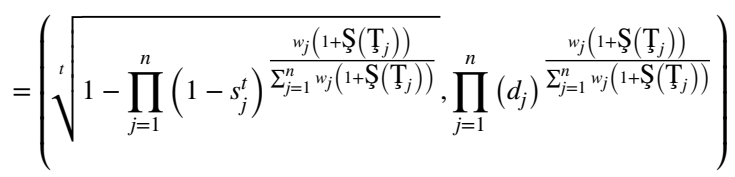

$\mathrm{q}-\operatorname{ROPFPWG}\left(\mathrm{T}_{1}, \mathrm{~T}_{2}, \mathrm{~T}_{3}, \ldots \mathrm{T}_{n}\right)$

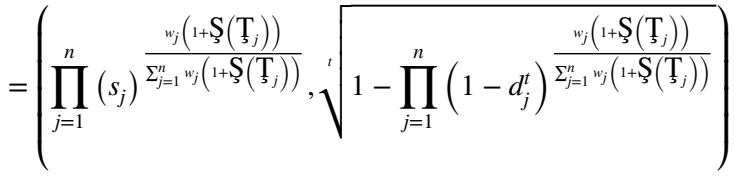

4. The Eqs. (7) and (10) results in the power WA and power WG aggregation operators of PyFSs proposed by Wei and Lu (2018) by taking $t=2$ and $u_{j}=0$ and given as:

$\operatorname{PyFPWA}\left(\mathrm{T}_{1}, \mathrm{~T}_{2}, \mathrm{~T}_{3}, \ldots \mathrm{T}_{n}\right)$

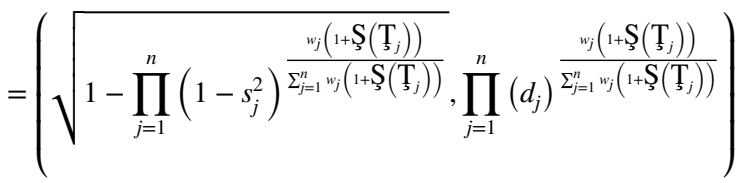

$$
\operatorname{PyFPWG}\left(\mathrm{T}_{1}, \mathrm{~T}_{2}, \mathrm{~T}_{3}, \ldots \mathrm{T}_{n}\right)
$$

$$
=\left(\prod_{j=1}^{n}\left(s_{j}\right)^{\frac{w_{j}\left(1+\mathbf{S}_{(j)}\left(\mathbf{T}_{j}\right)\right)}{\sum_{j=1}^{n} w_{j}\left(1+\mathbf{S}_{3}\left(\mathbf{T}_{j}\right)\right)}}, \sqrt{1-\prod_{j=1}^{n}\left(1-d_{j}^{2}\right)^{\frac{w_{j}\left(1+\mathbf{S}_{j}\left(\mathbf{T}_{j}\right)\right)}{\sum_{j=1}^{n} w_{j}\left(1+\mathbf{S}_{3}\left(\mathbf{T}_{j}\right)\right)}}}\right)
$$

5. The Eqs. (7) and (10) results in the power WA and power WG aggregation operators of IFSs proposed by $\mathrm{Xu}$ (2011) by taking $\mathrm{t}=1$ and $u_{j}=0$ and given as:
$\operatorname{IFPWA}\left(\mathrm{T}_{1}, \mathrm{~T}_{2}, \mathrm{~T}_{3}, \ldots \mathrm{T}_{n}\right)=\left(\begin{array}{c}1-\prod_{j=1}^{n}\left(1-s_{j}\right)^{\frac{w_{j=1}\left(1+\mathrm{S}_{j}\left(\mathrm{~T}_{j}\right)\right)}{\sum_{j}^{n}\left(1+\mathrm{S}_{3}\left(\mathrm{~T}_{j}\right)\right)}} \\ \prod_{j=1}^{n}\left(d_{j}\right)^{\frac{w_{j}\left(1+\mathrm{S}_{j}\left(\mathrm{~T}_{j}\right)\right)}{\sum_{j=1}^{n} w_{j}\left(1+\mathrm{S}_{3}\left(\mathrm{~T}_{j}\right)\right)}}\end{array}\right)$

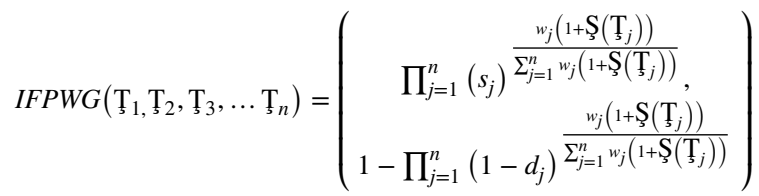

Here, Eqs. (15-24) provide the power WA and power WG operators for SFSs, PFSs and q-ROPFSs for which these types of aggregation operators are not defined yet. Equations (21-24) are special cases of TSFPWA and TSFPWG operators in which these operations reduced to the environment of PyFSs and IFSs studied in (Wei and Lu 2018; Xu 2011). All this clearly shows the significance and diverse structure of TSFPWA and TSFPWG operators.

The superiority of power aggregation operators of T-SFSs can be seen in the flowchart given in Fig. 2.

\section{Proposed multi-attribute decision making algorithm}

The aim of this section is to develop the algorithm for solving MADM problems using TSFPWA and TSFPWG operators. The process of MADM is briefly demonstrated followed by an algorithmic approach.

In MADM process, the selection of most suitable alternative is carried out under some attributes having weights and using the information provided by the decision makers. Suppose there are $n$ alternatives $\left(\mathcal{A}_{i}\right)$ with $m$ attributes $\left(g_{j}\right)$ having weight $w_{j}$. Further, let $d_{n \times m}=\left(\dot{\mathrm{T}}_{i j}\right)_{n \times m}=\left(s_{i j}, u_{i j}, d_{i j}\right)$ denote the decision matrix where $s_{i j}, u_{i j}$ and $d_{i j}$ are the degrees of satisfaction, abstinence and dissatisfaction respectively with $r=\sqrt[t]{1-\left(s_{i j}^{t}+u_{i j}^{t}+d_{i j}^{t}\right)}$ denote the refusal degree of alternative $\mathcal{A}_{i}$ 's under attributes $g_{j}$ 's. The following steps are followed for solving MADM problems based on the stated aggregation operators in the environment of T-SFNs.

Step 1: This first step involves the formation of decision matrix and investigation of data for finding the value of $t$ for which all the triplets becomes T-SFNs. 


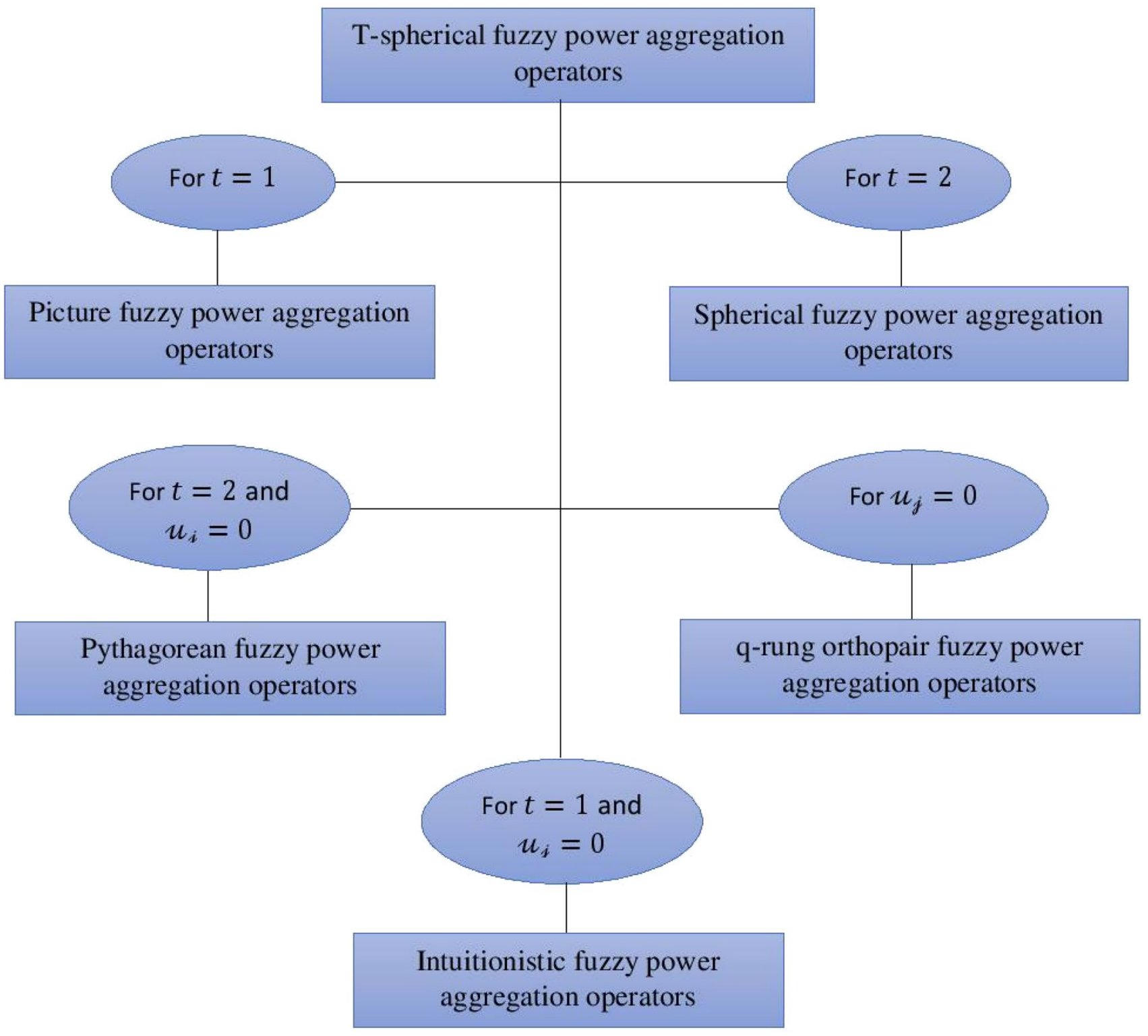

Fig. 2 Flowchart showing superiority of power aggregation operators of T-SFSs

Step 2: This step is based on the computation of support $\operatorname{Sup}\left(\mathrm{T}_{3}, \mathrm{~T}_{i k}\right)$ given by:

$\operatorname{Sup}\left(\mathrm{T}_{i j}, \mathrm{~T}_{i k}\right)=1-\mathbb{D}\left(\mathrm{T}_{i j}, \mathrm{~T}_{i k}\right)$

where $\mathbb{D}\left(\mathrm{T}_{i j}, \mathrm{~T}_{j k}\right)$ denote the Normalized Hamming distance proposed by Mahmood et al. (2018) given by:

$\mathbb{D}\left(\mathrm{T}_{i j}, \mathrm{~T}_{i k}\right)=\frac{1}{\mathrm{n}} \sum_{j, k=1}^{n}\left(\left|s_{i j}^{t}-s_{i k}^{t}\right|+\left|u_{i j}^{t}-u_{i k}^{t}\right|+\left|d_{i j}^{t}-d_{i k}^{t}\right|\right)$

Step 3: Compute the weighted support Ş( $\left.\mathrm{T}_{3 j}\right)$ of T-SFNs $\mathrm{T}_{i j}$ using the following formula given in Eq. (27)

$$
\mathrm{S}\left(\mathrm{T}_{i j}\right)=\sum_{\substack{i=1 \\ k \neq j}}^{n} w_{j} \operatorname{Sup}\left(\mathrm{T}_{i j}, \mathrm{~T}_{i k}\right)
$$

where $w_{j}$ is the weight vector of attributes $g_{j}$.

Step 4: Compute the weight $\delta_{i j}$ associated with T-SFNs $\mathrm{T}_{3 j}$ using Eq. (28)

$$
\delta_{i j}=\frac{w_{j}\left(1+\mathrm{S}_{3}\left(\mathrm{~T}_{i j}\right)\right)}{\sum_{j=1}^{n} w_{j}\left(1+\mathrm{S}_{3}\left(\mathrm{~T}_{i j}\right)\right)}
$$

$$
\text { where } \delta_{i j}>0 \text { such that } \sum_{j=1}^{n} \delta_{i j}=1 \text {. }
$$


Step 5: This step involves the aggregation of decision matrix $d$ using TSFPWA and TSFPWG operators given in Eqs. (29) and (30). screening based on their past records. The four alternatives are to be evaluated under four attributes $g_{j}(j=1,2,3,4)$ where $g_{1}, g_{2}, g_{3}$ and $g_{4}$ denote "Cost of information system",

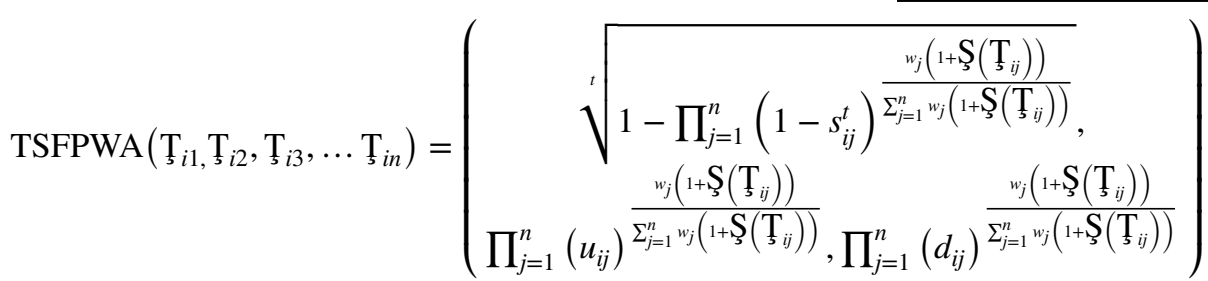

$\operatorname{TSFPWG}\left(\mathrm{T}_{i 1}, \mathrm{~T}_{i 2}, \mathrm{~T}_{i 3}, \ldots \mathrm{T}_{i n}\right)$

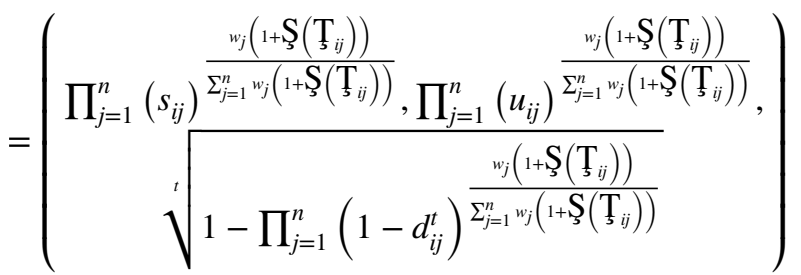

Step 6: Utilize the following score function to rank the alternatives based on the aggregated information obtained in Step 5.

Score $(\mathrm{T})=s^{t}-u^{t}-d^{t}$

Step 7: Based on the ranking of alternatives, the selection of best alternative is carried out.

\section{Numerical Example}

In this section, a numerical real-life problem is solved using the MADM method by utilizing the TSFPWA and TSFPWG aggregation operators for selection of best alternative.

Example 1 In this example, we consider the famous software selection problem in which the selection of a suitable software package is carried out among a list of software packages under some attributes. According to this problem, the university computer center needs to select an information system for a better research work production. For this case the computer center, along with HR department of the university, selected four alternatives $\mathcal{A}_{i}(i=1,2,3,4)$ after initial "involvement in performance of university", "Reliability of information system" and "efforts to transform from current system". The weight vector of these four attributes is $w=(0.3,0.25,0.25,0.2)^{\mathrm{T}}$. The decision makers critically observed the four alternatives based on the four attributes and presented their information in the form of T-SFNs in the following decision matrix given in Table 1 . This problem is solved using the MADM algorithm proposed in Sect. 5 and the detailed stepwise illustration of the process is as follows:

Step 1: The decision matrix containing the information about the four alternatives by anonymous decision makers.

Upon investigation, it is observed that the information provided in Table 1 cannot be considered as picture fuzzy numbers because their sum exceeds 1 . The sum of all membership grades of information in Table 1 is given is Table 2 (Table 3) .

It is observed that the information provided in Table 1 cannot be considered as spherical fuzzy numbers because the square of their sum exceeds 1 . The sum of squares of all elements of Table 1 is given Table 2.

The sum of all triplets of Table 1 is less than 1 if we take $t=3$ and are shown in Table 4.

All these observations clearly indicate that the aggregation tools of PFSs as well SFSs could not deal with such type

Table 2 Sum of all membership grades of elements provided in Table 1

\begin{tabular}{lrrrr}
\hline & $g_{1}$ & \multicolumn{2}{c}{$g_{2}$} & \multicolumn{2}{c}{$g_{3}$} & \multicolumn{2}{c}{$g_{4}$} \\
\hline $\mathcal{A}_{1}$ & 1.69 & 1.82 & 1.55 & 1.82 \\
$\mathcal{A}_{2}$ & 1.26 & 1.37 & 1.53 & 1.9 \\
$\mathcal{A}_{3}$ & 1.74 & 1.77 & 1.52 & 1.6 \\
$\mathcal{A}_{4}$ & 1.63 & 1.71 & 1.43 & 1.45 \\
\hline
\end{tabular}

Table 1 Decision matrix for selection of best information system

\begin{tabular}{lllll}
\hline & $g_{1}$ & $g_{2}$ & $g_{3}$ & $g_{4}$ \\
\hline $\mathcal{A}_{1}$ & $(0.34,0.64,0.71)$ & $(0.65,0.38,0.79)$ & $(0.41,0.59,0.55)$ & $(0.50,0.88,0.44)$ \\
$\mathcal{A}_{2}$ & $(0.80,0.11,0.35)$ & $(0.65,0.46,0.26)$ & $(0.91,0.41,0.21)$ & $(0.51,0.73,0.66)$ \\
$\mathcal{A}_{3}$ & $(0.73,0.58,0.43)$ & $(0.75,0.63,0.39)$ & $(0.49,0.39,0.64)$ & $(0.57,0.92,0.11)$ \\
$\mathcal{A}_{4}$ & $(0.54,0.32,0.77)$ & $(0.63,0.64,0.44)$ & $(0.46,0.80,0.17)$ & $(0.70,0.46,0.29)$ \\
\hline
\end{tabular}


Table 3 Sum of squares of all membership grades of elements provided in Table 1

\begin{tabular}{llllr}
\hline & $g_{1}$ & $g_{2}$ & $g_{3}$ & \multicolumn{1}{c}{$g_{4}$} \\
\hline $\mathcal{A}_{1}$ & 1.0293 & 1.191 & 0.8187 & 1.218 \\
$\mathcal{A}_{2}$ & 0.7746 & 0.7017 & 1.0403 & 1.2286 \\
$\mathcal{A}_{3}$ & 1.0542 & 1.1115 & 0.8018 & 1.1834 \\
$\mathcal{A}_{4}$ & 0.9869 & 1.0001 & 0.8805 & 0.7857 \\
\hline
\end{tabular}

Table 4 Sum of all elements of triplets provided in Table 1 for $t=3$

\begin{tabular}{lllll}
\hline & $g_{1}$ & $g_{2}$ & $g_{3}$ & \multicolumn{2}{l}{$g_{4}$} \\
\hline $\mathcal{A}_{1}$ & 0.659359 & 0.822536 & 0.440675 & 0.891656 \\
$\mathcal{A}_{2}$ & 0.556206 & 0.389537 & 0.831753 & 0.809164 \\
$\mathcal{A}_{3}$ & 0.663636 & 0.731241 & 0.439112 & 0.965212 \\
$\mathcal{A}_{4}$ & 0.646765 & 0.597375 & 0.614249 & 0.464725 \\
\hline
\end{tabular}

of information. Further, T-SFNs involve four membership grades and hence cannot be aggregated by the aggregation tools of q-ROPFSs, PyFSs as well as IFSs. This shows that existing aggregation tools are unable to deal with T-spherical fuzzy information.

Using Eqs. (25-28) discussed in Steps 2, 3 and 4, we found the value of $\delta_{i j}$ given by:

$\delta_{i j}=(0.311573,250135,247281,191012)^{\mathrm{T}}$

where

$\delta_{i j}=\frac{w_{j}\left(1+\mathrm{S}_{j}\left(\mathrm{~T}_{i j}\right)\right)}{\sum_{j=1}^{n} w_{j}\left(1+\mathrm{S}_{s}\left(\mathrm{~T}_{i j}\right)\right)}$

Step 5: Utilizing Eqs. $(29,30)$ and $\delta_{i j}$ obtained in Steps $2-5$, we aggregate the information of decision makers given in Table 1 and the aggregated data using TSFPWA and TSFPWG operators is given in Table 5.

Step 6: Utilizing Eq. (31) to get the score values of data obtained in Table 6.

Step 7: Based on the score values obtained in Step 6, the four alternatives are ranked in Table 7

The ranking of alternatives using TSFPWA and TSFPWG operators in Table 7 gives us two possible best alternatives. According to TSFPWA operator, $\mathcal{A}_{2}$ is the best option while if we consider TSFPWG operator then it comes out that $\mathcal{A}_{4}$ is the best option. The selection of aggregation tool is up to decision makers.
Table 5 Aggregated data using Eqs. (29) and (30)

\begin{tabular}{lll}
\hline & TSFPWA operator & TSFPWG operator \\
\hline $\mathcal{A}_{1}$ & $(0.5028,0.5851,0.6248)$ & $(0.4508,0.5851,0.6762)$ \\
$\mathcal{A}_{2}$ & $(0.7895,0.714,0.3232)$ & $(0.8350,0.7143,0.2570)$ \\
$\mathcal{A}_{3}$ & $(0.6715,0.5862,0.3568)$ & $(0.8757,0.5862,0.3163)$ \\
$\mathcal{A}_{4}$ & $(0.5908,0.512,0.3824)$ & $(0.8890,0.5116,0.4791)$ \\
\hline
\end{tabular}

Table 6 Score values of alternatives

\begin{tabular}{lll}
\hline Scores & TSFPWA operator & TSFPWG operator \\
\hline $\mathcal{A}_{1}$ & -0.31708 & -0.41794 \\
$\mathcal{A}_{2}$ & 0.09386 & 0.200728 \\
$\mathcal{A}_{3}$ & 0.055878 & 0.438338 \\
$\mathcal{A}_{4}$ & 0.016373 & 0.458634 \\
\hline
\end{tabular}

Table 7 Ranking of alternatives

\begin{tabular}{ll}
\hline Operators & Ranking \\
\hline TSFPWAoperator & $\mathcal{A}_{2}>\mathcal{A}_{3}>\mathcal{A}_{4}>\mathcal{A}_{1}$ \\
TSFPWGoperator & $\mathcal{A}_{4}>\mathcal{A}_{3}>\mathcal{A}_{2}>\mathcal{A}_{1}$ \\
\hline
\end{tabular}

\section{Comparative study}

In this section, the comparative study of TSFPWA and TSFPWG operators is established with power aggregation operators of IFSs and PyFSs. From our study we claim that the pre-existing power aggregation operators in the environment of PyFSs and IFSs cannot handle the data provided in the form of T-SFNs. This is clearly shown in Sect. 6, Example 1. On the other hand, we show that the proposed TSFPWA and TSFPWG aggregation operators can solve the problems having information in the form of q-ROPFSs, PyFSs as well as IFSs.

Example 2 We consider the MADM problem discussed in Sect. 6 and drop the abstinence degree. In this way, the data provided in Table 1 will be reduced to $\mathrm{q}-\mathrm{ROPFNs}$ for $\mathrm{q}=3$ and is given in Table 8 below. The weight vector of these four attributes is same i.e. $w=(0.3,0.25,0.25,0.2)^{\mathrm{T}}$.

We utilized q-ROPFPWA and q-ROPFPWG operators by following the algorithm proposed in Sect. 5 and the results are listed in Tables 8, 9, 10 respectively. 
Table 8 Decision matrix obtained by considering abstinence degree as zero

\begin{tabular}{lllll}
\hline & $g_{1}$ & $g_{2}$ & $g_{3}$ & $g_{4}$ \\
\hline $\mathcal{A}_{1}$ & $(0.34,0.71)$ & $(0.65,0.79)$ & $(0.41,0.55)$ & $(0.50,0.44)$ \\
$\mathcal{A}_{2}$ & $(0.80,0.35)$ & $(0.65,0.26)$ & $(0.91,0.21)$ & $(0.51,0.66)$ \\
$\mathcal{A}_{3}$ & $(0.73,0.43)$ & $(0.75,0.39)$ & $(0.49,0.64)$ & $(0.57,0.11)$ \\
$\mathcal{A}_{4}$ & $(0.54,0.77)$ & $(0.63,0.44)$ & $(0.46,0.17)$ & $(0.70,0.29)$ \\
$\mathcal{A}_{5}$ & $(0.34,0.71)$ & $(0.65,0.79)$ & $(0.41,0.55)$ & $(0.50,0.44)$ \\
\hline
\end{tabular}

Table 9 Aggregated data using Eqs. (19) and (20)

\begin{tabular}{lll}
\hline & q - ROPFPWA operator & q - ROPFPWG operator \\
\hline $\mathcal{A}_{1}$ & $(0.502583,0.624884)$ & $(0.450547,0.676221)$ \\
$\mathcal{A}_{2}$ & $(0.78974,0.323079)$ & $(0.834949,0.256904)$ \\
$\mathcal{A}_{3}$ & $(0.671476,0.357268)$ & $(0.875694,0.316338)$ \\
$\mathcal{A}_{4}$ & $(0.590576,0.382433)$ & $(0.889338,0.478529)$ \\
\hline
\end{tabular}

Table 10 Score values of alternatives

\begin{tabular}{lll}
\hline Score of & q-ROPFPWA operator & q-ROPFPWG operator \\
\hline $\mathcal{A}_{1}$ & -0.11706 & -0.21776 \\
$\mathcal{A}_{2}$ & 0.458829 & 0.56512 \\
$\mathcal{A}_{3}$ & 0.257153 & 0.639861 \\
$\mathcal{A}_{4}$ & 0.150049 & 0.593818 \\
\hline
\end{tabular}

Table 11 Ranking of alternatives

\begin{tabular}{ll}
\hline Operators & Ranking \\
\hline$q-$ ROPFPWAoperator & $\mathcal{A}_{2}>\mathcal{A}_{3}>\mathcal{A}_{4}>\mathcal{A}_{1}$ \\
$q-$ ROPFPWGoperator & $\mathcal{A}_{3}>\mathcal{A}_{4}>\mathcal{A}_{2}>\mathcal{A}_{1}$ \\
\hline
\end{tabular}

Using Eqs. (25-28) discussed in Steps 2, 3 and 4, we found the value of $\delta_{i j}$ given by:

$\delta_{i j}=(0.312311,0.249562,0.247797,0.190329)^{\mathrm{T}}$

Step 5: Utilizing Eqs. $(19,20)$ and $\delta_{i j}$, we aggregate the information of decision makers given in Table 8 and the aggregated data using q-ROPFPWA and q-ROPFPWG operators is given in Table 9.

Step 6: Utilizing Eq. (31) to get the score values of data obtained in Table 10.

Step 7: Based on the score values obtained in Step 6, the four alternatives are ranked in Table 11.

The ranking of alternatives using q-ROPFPWA and q-ROPFPWG operators in Table 11 gives us two possible best alternatives which are totally different from the ranking obtained using TSFPWA and TSFPWG operators given in
Table 7. This shows the importance of using T-SFSs over of other fuzzy frameworks such as IFSs, PyFSs, q-ROPFSs and PFSs.

Here the same problem is considered with same data as well. The only difference is that in Example 1 degree of abstinence is considered while in Example 2 the degree of abstinence is neglected. Therefore, the results obtained in Example 1 are more reliable than that of obtained in Example 2. This shows the superiority of T-SFSs and consequently T-spherical fuzzy power aggregation operators over the existing related tools.

The advantages of $\mathrm{T}$-spherical fuzzy power aggregation operators are as follows:

1. T-spherical fuzzy power aggregation operators take into account the relationship of the information being used (aggregated) however the previous aggregation operators fail to do so.

2. T-spherical fuzzy power aggregation operators take into account the abstinence and refusal degree while power aggregation operators of IFSs, PyFSs and q-ROFSs consider only membership and non-membership grades. This inclusion of abstinence and refusal grade of membership improves the results and decrease information loss as shown in Example 1 and 2.

3. While using T-spherical fuzzy power aggregation operators, the decision makers have a larger range for assigning membership grades due the parameter $t$ unlike in case of picture fuzzy power aggregation operators or spherical fuzzy power aggregation operators.

\section{Conclusion}

The key contribution of the work can be summarized below.

1. A concept of T-spherical fuzzy set has been utilized to describe the uncertainties in the data. A T-SFS utilize four functions to describe the imprecision of an event including favor, abstinence, and disfavor and refusal degrees. From the geometry of T-SFS (given in Fig. 1), it is observed that it has wider range of information as compared to existing IFS, PyFS, PFS, SFS and q-ROFS. Thus, a concept of T-SFS affords an alternative way to trade with the uncertainties.

2. A T-SFS is an extension of several existing sets (see Remark 1) and hence by setting a parameter $t$, we can deduce such existing sets and their corresponding studies from the presented work.

3. In the work, we stated several power weighted aggregation operators namely, T-spherical fuzzy power weighted, ordered weighted and hybrid average operators. Several desirable relations have been derived to 
study their properties. Further, we extend them to geometric operators also and study their features. The major advantage of using such power aggregation operators is that it takes into account the relationship of the information being aggregated as compared to several other existing operators (Garg and Kumar 2020; Wang et al. 2020; Yager 2001; Xu and Yager 2010; Wei et al. 2013; Wang and Li 2020; Wei and Lu 2018; Garg and Arora 2018, 2019; Xu 2011).

4. The MADM algorithm based on the stated operators is explained, which is more generalized and flexible with the parameter $t$ to the decision-maker. The applicability of the algorithm is demonstrated through a numerical example (in Example 1). Compared with the approaches stated in (Garg and Kumar 2020; Wang et al. 2020; Yager 2001; Xu and Yager 2010; Wei et al. 2013; Wang and Li 2020; Wei and Lu 2018; Garg and Arora 2018, 2019; Xu 2011), our approach is more suitable and widely applicable to solve the MADM problem under the diverse fuzzy environment.

In the future, we will expand our study and able to tackle the real-problems under the different fuzzy environment (Garg 2020a; Arora and Garg 2018; Akram et al. 2020; Nancy and Garg 2019).

\section{Compliance with ethical standards}

Conflict of interest The authors declare that they have no conflicts of interest regarding the publication of this article.

\section{References}

Akram M, Garg H, Zahid K (2020) Extensions of ELECTRE-I and TOPSIS methods for group decision-making under complex Pythagorean fuzzy environment. Iran. J. Fuzzy Syst. 17(5):147-164

Arora R, Garg H (2018) A robust correlation coefficient measure of dual hesistant fuzzy soft sets and their application in decision making. Eng Appl Artif Intell 72:80-92

Atanassov K, Gargov G (1989) Interval-valued intuitionistic fuzzy sets. Fuzzy Sets Syst 31:343-349

Atanassov KT (1986) Intuitionistic fuzzy sets. Fuzzy Sets Syst 20(1):87-96

Cuong BC (2014) Picture fuzzy sets. J Comput Sci Cybern 30(4):409

Garg H (2017a) Generalized Pythagorean fuzzy geometric aggregation operators using Einstein t-norm and t-conorm for multicriteria decision-making process. Int J Intell Syst 32(6):597-630

Garg H (2017b) Some picture fuzzy aggregation operators and their applications to multicriteria decision-making. Arab J Sci Eng 42(12):5275-5290

Garg H (2020a) Exponential operational laws and new aggregation operators for intuitionistic multiplicative set in multiple-attribute group decision making process. Inf Sci 538:245-272

Garg H (2020b) Linguistic interval-valued Pythagorean fuzzy sets and their application to multiple attribute group decision-making process. Cognit Comput. https://doi.org/10.1007/s12559-02009750-4

Garg H, Arora R (2018) Novel scaled prioritized intuitionistic fuzzy soft interaction averaging aggregation operators and their application to multi criteria decision making. Eng Appl Artif Intell 71C:100-112

Garg H, Arora R (2019) Generalized intuitionistic fuzzy soft power aggregation operator based on t-norm and their application in multi criteria decision-making. Int J Intell Syst 34(2):215-246

Garg H, Kaur G (2019) Cubic intuitionistic fuzzy sets and its fundamental properties J. Multiple-Valued Logic Soft Comput 33(6):507-537

Garg H, Kumar K (2019) Linguistic interval-valued Atanassov intuitionistic fuzzy sets and their applications to group decision-making problems. IEEE Trans Fuzzy Syst 27(12):2302-2311

Garg H, Kumar K (2020) Power geometric aggregation operators based on connection number of set pair analysis under intuitionistic fuzzy environment. Arab J Sci Eng 45(3):2049-2063

Garg H, Munir M, Ullah K, Mahmood T, Jan N (2018) Algorithm for T-spherical fuzzy multi-attribute decision making based on improved interactive aggregation operators. Symmetry 10(12):670. https://doi.org/10.3390/sym10120670

Kaur G, Garg H (2019) Generalized cubic intuitionistic fuzzy aggregation operators using t-norm operations and their applications to group decision-making process. Arab J Sci Eng 44(3):2775-2794

Liu P, Khan Q, Mahmood T, Hassan N (2019) T-spherical fuzzy power Muirhead mean operator based on novel operational laws and their application in multi-attribute group decision making. IEEE Access 7:22613-22632

Liu P, Wang P (2018) Some q-rung orthopair fuzzy aggregation operators and their applications to multiple-attribute decision making. Int J Intell Syst 33(2):259-280

Mahmood T, Ullah K, Khan Q, Jan N (2018) An approach toward decision-making and medical diagnosis problems using the concept of spherical fuzzy sets. Neural Comput Appl 31:7041-7053

Munir M, Kalsoom H, Ullah K, Mahmood T, Chu Y-M (2020) T-spherical fuzzy Einstein hybrid aggregation operators and their applications in multi-attribute decision making problems. Symmetry 12(3):365

Nancy and Garg, H. (2019) A novel divergence measure and its based TOPSIS method for multi criteria decision - making under single - valued neutrosophic environment. J Intell Fuzzy Syst 36(1):101-115

Peng X, Yang Y (2015) Some results for Pythagorean fuzzy sets. Int J Intell Syst 30(11):1133-1160

Rani D, Garg H (2018) Complex intuitionistic fuzzy power aggregation operators and their applications in multi-criteria decision-making. Expert Syst 35(6):e12325

Ren PJ, Xu ZS, Gou XJ (2016) Pythagorean fuzzy TODIM approach to multi-criteria decision making. Appl Soft Comput 42:246-259

Ullah K, Garg H, Mahmood T, Jan N, Ali Z (2020) Correlation coefficients for T-spherical fuzzy sets and their applications in clustering and multi-attribute decision making. Soft Comput 24(3):1647-1659

Ullah K, Hassan N, Mahmood T, Jan N, Hassan M (2019) Evaluation of investment policy based on multi-attribute decision-making using interval valued T-spherical fuzzy aggregation operators. Symmetry 11(3):357

Ullah K, Mahmood T, Garg H (2020) Evaluation of the performance of search and rescue robots using T-spherical fuzzy Hamacher aggregation operators. Int J Fuzzy Syst 22(2):570-582

Ullah K, Mahmood T, Jan N (2018) Similarity measures for t-spherical fuzzy sets with applications in pattern recognition. Symmetry 10(6): 193

Wang C, Zhou X, Tu H, Tao S (2017) Some geometric aggregation operators based on picture fuzzy sets and their application 
in multiple attribute decision making. Ital J Pure Appl Math 37:477-492

Wang L, Garg H, Li N (2020) Pythagorean fuzzy interactive Hamacher power aggregation operators for assessment of express service quality with entropy weights. Soft Comput 1-21. https://doi. org/10.1007/s00500-020-05193-z

Wang L, Li N (2020) Pythagorean fuzzy interaction power Bonferroni mean aggregation operators in multiple attribute decision making. Int J Intell Syst 35(1):150-183

Wei G, Zhang Z (2019) Some single-valued neutrosophic Bonferroni power aggregation operators in multiple attribute decision making. J Ambient Intell Human Comput 10:863-882

Wei G, Zhao X, Wang H, Lin R (2013) Fuzzy power aggregation operators and their application to multiple attribute group decision making. Technol Econ Dev Econ 19(3):377-396

Wei GW, Lu M (2018) Pythagorean fuzzy power aggregation operators in multiple attribute decision maig. Int J Intell Syst 33(1):169-186

$\mathrm{Xu} \mathrm{Z} \mathrm{(2011)} \mathrm{Approaches} \mathrm{to} \mathrm{multiple} \mathrm{attribute} \mathrm{group} \mathrm{decision} \mathrm{making}$ based on intuitionistic fuzzy power aggregation operators. KnowlBased Syst 24(6):749-760

Xu Z, Yager RR (2010) Power - geometric operators and their use in group decision making. IEEE Trans Fuzzy Syst 18(1):94-105

Xu ZS (2007) Intuitionistic fuzzy aggregation operators. IEEE Trans Fuzzy Syst 15:1179-1187

Xu ZS, Yager RR (2006) Some geometric aggregation operators based on intuitionistic fuzzy sets. Int J Gen Syst 35:417-433
Yager RR (2001) The power average operator. IEEE Syst Man Cybern Soc 31(6):724-731

Yager RR (2013) Pythagorean fuzzy subsets. In: Proceedings joint IFSA world congress and NAFIPS annual meeting, Edmonton, pp 57-61

Yager RR (2017) Generalized orthopair fuzzy sets. IEEE Trans Fuzzy Syst 25(5):1222-1230

Yang Z, Garg H, Li J, Srivastava G, Cao Z (2020) Investigation of multiple heterogeneous relationships using a q-rung orthopair fuzzy multi-criteria decision algorithm. Neural Comput Appl 1-22. https://doi.org/10.1007/s00521-020-05003-5

Yang Z, Li X, Garg H, Qi M (2020) Decision support algorithm for selecting an antivirus mask over COVID-19 pandemic under spherical normal fuzzy environment. Int J Environ Res Public Health 17(10):3407

Yang Z, Ouyang T, Fu X, Peng X (2020) A decision-making algorithm for online shopping using deep-learning-based opinion pairs mining and q-rung orthopair fuzzy interaction Heronian mean operators. Int J Intell Syst 35(5):783-825

Zadeh LA (1965) Fuzzy sets. Inf Control 8:338-353

Zhou L, Chen H, Lu J (2012) Generalized power aggregation operators and their applications in group decision making. Comput Ind Eng 62(4):989-999

Publisher's note Springer Nature remains neutral with regard to jurisdictional claims in published maps and institutional affiliations. 